\title{
Percepção e conhecimento do enfermeiro frente ao tratamento conservador da
}

\section{doença renal}

\author{
Perception and knowledge of the nurse in front of the conservative treatment of kidney disease \\ Percepción y conocimiento de la enfermeira frente al tratamento conservador de la enfermedad \\ renal
}

Recebido: 21/01/2021 | Revisado: 23/01/2021 | Aceito: 27/01/2021 | Publicado: 04/02/2021

\author{
Ananda Olívio Camargo \\ ORCID: https://orcid.org/0000-0003-0338-5500 \\ Centro Universitário da Fundação Hermínio Ometto, Brasil \\ E-mail: nandaacamargo@hotmail.com \\ Tainá Emanuelle Coelho Rebelo \\ ORCID: https://orcid.org/0000-0003-3680-6236 \\ Centro Universitário da Fundação Hermínio Ometto, Brasil \\ E-mail: tahtinha87@gmail.com \\ Juliana Furlan Ravagnani \\ ORCID: https://orcid.org/0000-0002-2058-1258 \\ Hospital das Clínicas da Universidade Estadual de Campinas, Brasil \\ E-mail:jravagnani@hc.unicamp.br \\ Aline Scharr Rodrigues \\ ORCID: https://orcid.org/0000-0002-8336-6037 \\ Universidade de São Paulo, Brasil \\ E-mail:alinescharr@usp.br \\ Clarice Santana Milagres \\ ORCID: https://orcid.org/0000-0002-9164-4340 \\ Centro Universitário da Fundação Hermínio Ometto, Brasil \\ E-mail:claricemilagres01@gmail.com
}

\begin{abstract}
Resumo
O tratamento conservador do paciente renal se faz necessário em todas as instâncias da progressão da doença renal, visto o aumento exponencial de pacientes em terapia dialítica. O enfermeiro possui papel chave no manejo da doença, sendo necessário o conhecimento acerca de todas as formas de tratamento, das terapias de tratamento e dos cuidados específicos que o paciente precisa. Objetivo: verificar o conhecimento dos enfermeiros atuantes na assistência aos pacientes com progressão de perda da função renal, sobre as terapias dialíticas, assim como identificar suas percepções sobre o tratamento conservador em uma unidade de internação hospitalar. Metodologia: estudo de abordagem qualitativa em um hospital universitário no interior do Estado de São Paulo. A população do estudo foram enfermeiros que atuavam na Unidade de Internação Adulto. Foi realizada uma entrevista semiestruturada com questões discursivas, analisadas segundo o discurso do sujeito coletivo de Bardin. Resultados: dez enfermeiros compuseram a pesquisa e a partir da análise das transcrições das entrevistas emergiram duas categorias: conhecimento do enfermeiro sobre as terapias dialíticas e conhecimento e percepção do enfermeiro sobre o tratamento conservador. Conclusão: Contempla-se que há o conhecimento a respeito das terapias dialíticas e do tratamento conservador pelos enfermeiros. Contudo ressalta-se a importância da educação continuada de todos os setores intra hospitalares sobre o tratamento dialítico e conservador do paciente renal.
\end{abstract}

Palavras-chave: Doença renal crônica; Unidades de internação; Educação continuada.

\begin{abstract}
Conservative treatment of renal patients is necessary in all instances of kidney disease progression, given the exponential increase in patients undergoing dialysis therapy. The nurse has a key role in the management of the disease, requiring knowledge about all forms of treatment, treatment therapies and specific care that the patient needs. Objective: to verify the knowledge of nurses, active in assisting patients with progression of renal function loss, on dialysis therapies, as well as to identify their perceptions about conservative treatment in a hospitalization unit. Methodology: study of a qualitative approach in a university hospital in the state of São Paulo. The study population was nurses who worked in the Adult Inpatient Unit. A semi-structured interview was conducted with discursive questions, analyzed according to the discourse of the collective subject of Bardin. Results: ten nurses composed the research and from the analysis of the interview transcripts, two categories emerged: nurses 'knowledge about dialysis therapies and nurses' knowledge and perception about conservative treatment. Conclusion: It is contemplated that nurses have knowledge about dialysis
\end{abstract}


therapies and conservative treatment. However, the importance of continuing education in all intra-hospital sectors on dialysis and conservative treatment of renal patients is emphasized.

Keywords: Renal insufficiency chronic; Inpatient care units-hospital; Continuing education.

\section{Resumen}

El tratamiento conservador de los pacientes renales es necesario en todos los casos de progresión de la enfermedad renal, dado el aumento exponencial de los pacientes sometidos a diálisis. La enfermera tiene un papel clave en el manejo de la enfermedad, requiriendo conocimiento sobre todas las formas de tratamiento, terapias de tratamiento y cuidados específicos que el paciente necesita. Objetivo: verificar el conocimiento de enfermeras, activas en la asistencia a pacientes con progresión de pérdida de función renal, sobre terapias de diálisis, así como identificar sus percepciones sobre el tratamiento conservador en una unidad de hospitalización. Metodología: estudio de abordaje cualitativo en un hospital universitario del estado de São Paulo. La población de estudio fueron enfermeras que laboraban en la Unidad de Adultos Hospitalizados. Se realizó una entrevista semiestructurada con preguntas discursivas, analizadas según el discurso del sujeto colectivo de Bardin. Resultados: diez enfermeras compusieron la investigación y del análisis de las transcripciones de las entrevistas surgieron dos categorías: el conocimiento de las enfermeras sobre las terapias de diálisis y el conocimiento y percepción de las enfermeras sobre el tratamiento conservador. Conclusión: Se contempla que los enfermeros tengan conocimientos sobre terapias de diálisis y tratamiento conservador. No obstante, se destaca la importancia de la formación continua en todos los sectores intrahospitalarios sobre diálisis y tratamiento conservador de los pacientes renales.

Palabras clave: Insuficiencia renal crónica; Unidades de internación; Educación continua.

\section{Introdução}

As transformações demográficas têm sido influenciadas diretamente pela queda da fertilidade, mortalidade infantil e aumento da expectativa de vida. Somadas às modificações nos padrões alimentares e à redução da atividade física, a transição epidemiológica também é observada junto ao crescente índice de doenças crônicas degenerativas, das quais a Doença Renal Crônica (DRC) apresenta importante incidência em nível mundial (Pereira, Chemin, Menegatti \& Riella, 2016).

Considerada como problema de saúde mundial, a DRC afeta aproximadamente 13\% da população adulta e estima-se que o custo anual do tratamento dialítico ultrapasse 1,5 bilhões (Pereira, et al., 2016; Gonçalves, Dalosso, Borba, Bucaneve, Valerio, Okamoto \& Bucharles, 2015). No Brasil, essa doença tem aumentado consideravelmente e estima-se que a incidência de novos pacientes em diálise, por ano, esteja em torno de 42.500, com tendências para aumentar. Segundo censo de diálise da Sociedade Brasileira de Nefrologia (SBN), em relação às terapias renais substitutivas, o ano de 2019 apresentou 93,2\% dos pacientes em hemodiálise (HD) e apenas 6,8\% em diálise peritoneal (Neves, Sesso, Thomé, Lugon \& Nascimento, 2020; Thomé, Sesso, Lopes, Lugon \& Martins, 2019).

A DRC progride de forma lenta e gradual com diversos sinais e sintomas decorrentes da incapacidade do rim de manter a homeostase do organismo. A perda irreversível e progressiva da função renal, que caracteriza a sua cronicidade, é dividida em leve, moderada, grave ou terminal. Segundo o Kidney Disease Outcome Quality Iniciative (KDOQI) este estadiamento, classifica em estágios progressivos do 1 (mais leve) ao 5 (mais grave), de acordo com a Taxa de Filtração Glomerular (TFG). Logo, dos cinco estágios evolutivos da DRC, é no último estágio que se decorre a necessidade do tratamento através da Terapia Renal Substitutiva (TRS) ou transplante renal (Daugirdas, Blake \& Todd, 2016).

A TRS apresenta diferentes formas de ser realizada: diálise peritoneal (DP) e hemodiálise. A DP é um método que possui baixa penetração no cenário mundial, necessitando, portanto, da melhor compreensão de fatores contribuintes para seu crescimento, uma vez que oferece níveis de efetividade e segurança semelhantes à hemodiálise. Contudo, a preferência do tratamento dialítico por HD ainda é superior à DP. No Brasil, a hemodiálise é terapia dialítica adotada com maior predominância entre a última década (aproximadamente 92\%), enquanto a DP apresenta uma redução progressiva (10,5-7,8\%) de seu uso no mesmo período. Contudo, a literatura internacional aponta que aproximadamente 12 a 305 dos pacientes preferem a DP à HD (Neves, et al., 2020; Daugirdas, Blake \& Todd (2016). Na DP, a filtração das impurezas do sangue é feita pelo peritônio. Os pacientes são devidamente treinados para realizarem o tratamento de forma autônoma e sem necessidade de equipamentos de 
alta tecnologia e complexidade que são oferecidos em clínicas de diálise (Pereira, Batista, Meira, Oliveira \& Kusumota, 2017; Pereira, et al., 2016; Moura Neto, Souza, Moura, Oliveira, Paschoalim, Paschoalim \& Moura Júnior, 2014).

O tratamento conservador na DRC, consiste em medidas clínicas, como prescrição medicamentosa, dieta controlada e restritiva, e modificações de estilo de vida (Bastos \& Kirsztajn, 2011). Estas medidas podem ser utilizadas para retardar a progressão da doença, por auxiliar a reduzir os sintomas clássicos, assim como, possibilitam prevenir eventuais complicações. Este tratamento apresenta como propósito fornecer ao portador de DRC um bom estado clínico, conciliável com a fase da doença em que se encontra e é frequentemente utilizado a partir do momento do diagnóstico da doença e mantido a longo prazo (Xavier, Hermógenes, Ribeiro, Sá, Ávila \& Flores, 2020; Pereira, et al., 2017). O tratamento conservador apresenta impacto positivo na sobrevida e na qualidade de vida desses pacientes, principalmente se iniciado precocemente, uma vez que há uma chance maior de preservar a função renal por um período mais extenso de tempo (Xavier, et al., 2020).

A abordagem do paciente com DRC em tratamento conservador e em preparação para diálise abrange diversas etapas, como: rastreamento, diagnóstico etiológico, estadiamento da doença, manejo do alto risco de progressão, identificação das complicações e preparação do paciente para transplante preemptivo ou TRS (Daugirdas, Blake \& Todd, 2016). O conhecimento do enfermeiro, responsável pela equipe e pelos cuidados ao portador de doença renal em progressão, faz-se necessário ao contribuir para o empoderamento e adaptações necessárias que serão implantadas na rotina destes indivíduos. Busca-se assim, promover maior flexibilidade no tratamento, melhor vida social e normalização da rotina criada após a descoberta da doença (Fernandes, Zanelli, Scharr Rodrigues, Rodrigues, Lodi, Marques, Reis, Ferreira \& Milagres, 2020).

Ressalta-se que o enfermeiro é um profissional capaz de proporcionar, através da educação em saúde, o autocuidado ao portador de doença renal em tratamento conservador. Quando consciente e disciplinado acerca deste tratamento, o paciente pode percebê-lo de forma positiva, contribuindo, portanto, para alcance de melhor qualidade de vida, redução de complicações físicas, mentais e sociais, além de reduzir a progressão da perda renal (Fernandes, et al., 2020).

Diante do exposto, analisar como o enfermeiro está inserido no tratamento conservador da doença renal e qual a sua percepção sobre tal tratamento se faz necessário. Assim, este estudo teve como objetivo verificar o conhecimento de enfermeiros atuantes na assistência ao portador de doença renal crônica fora da unidade de internação adulto, analisando a percepção destes acerca do tratamento conservados da doença renal.

\section{Metodologia}

\section{Design e período}

Trata-se de uma pesquisa descritiva, exploratória, de natureza qualitativa, desenvolvida no Hospital das Clínicas da Universidade Estadual de Campinas (UNICAMP), no interior do Estado de São Paulo, Brasil. Para Pereira, Shitsuka, Parreira \& Shitsuka (2018), os métodos qualitativos apresentam a interpretação do pesquisador como parte importante do processo, uma vez que são percebidas e apresentadas suas opiniões sobre o fenômeno em estudo e muitas vezes, a coleta de dados ocorre por meio de entrevistas com questões abertas. Para a realização deste estudo, foram adotados os critérios do Consolidated Criteria for Reporting Qualitative Research (Tong, Sainsbury \& Craig, 2007).

Os dados foram coletados de fevereiro a abril de 2019, com enfermeiros colaboradores da Unidade de Internação Adulto (UIA).

\section{Coleta de Dados}

A coleta de dados ocorreu em sessão única, com aproximadamente 60 minutos de duração. Decorreu-se a partir da assinatura do Termo de Consentimento Livre e Esclarecido (TCLE), seguida da aplicação dos questionários de identificação e questões semi estruturadas relativas à temática proposta. 
A opção pelo questionário é devida à possibilidade de atingir um maior número de enfermeiros, através da obtenção de respostas rápidas e precisas. Também há maior segurança para responder, pois não há identificação dos sujeitos que aceitam participar da pesquisa, além de não haver interferência do pesquisador durante a condução da aplicação do questionário.

\section{Amostra, critérios de inclusão e exclusão}

A amostra foi composta por enfermeiros colaboradores do Hospital das Clínicas da Universidade Estadual de Campinas (UNICAMP), na Unidade de Internação Adulto (UIA). Os critérios de inclusão estabelecidos foram: idade maior ou igual a 18 anos, graduado em enfermagem, ter experiência clínica com pacientes renais, concordar e autorizar a participação da pesquisa assinando o TCLE. Foram excluídas da amostra aqueles enfermeiros que não atenderam aos critérios de inclusão, recusaram participar ou retiraram o seu consentimento.

\section{Variáveis de estudo}

Os dados foram coletados por duas pesquisadoras que realizaram um contato prévio com os enfermeiros da UIA, no qual foi apresentada e esclarecida a pesquisa, assim como foi obtida a assinatura no TCLE dos entrevistados. A entrevista seguiu um roteiro semiestruturado com questões como: idade autodeclarada, tempo de formação em enfermagem, instituição de formação de graduação em enfermagem, obtenção (ou não) de título de enfermeiro nefrologista.

As demais informações abordaram: o conhecimento sobre tratamentos para a DRC, entendimento acerca da DRC e tratamento conservador, oportunidade de vivenciar o tratamento conservador em DRC, conhecimento acerca do tratamento conservador da doença renal, sentimentos acerca da necessidade de ter que realizar o tratamento conservador em um portador de doença renal, percepção acerca da aceitação (ou não) do paciente frente ao tratamento conservador e forma de implantação deste tipo de tratamento em pacientes portadores de doença renal.

\section{Metodologia de Análise De Dados}

A análise dos dados foi realizada por meio da Análise de Conteúdo de Bardin que procura compreender os objetivos dos estudos desenvolvidos. É um meio de expressão do sujeito, no qual o analista busca categorizar as unidades de texto (palavras ou frases) que se repetem, inferindo uma expressão que as representem. Este tipo de análise apresenta uma técnica capaz de proporcionar uma avaliação organizada do texto, ao identificar os temas mais recorrentes e as palavras mais relevantes para ser investigado. Posteriormente, compara-se as informações coletadas a fim de se obter uma conclusão, que se dá por um tratamento da informação contida nas mensagens dos participantes (Bardin, 2009).

A metodologia proposta foi a categorização, inserida na análise de conteúdo, na qual emprega a representatividade, ou seja, uma amostra de representação de um universo inicial. Para as três etapas da categorização da análise de conteúdo foram observadas:

- Pré-análise, que consistiu na escolha dos documentos a serem analisados, sistematização de hipóteses e ideias iniciais e criação de categorias de análise;

- Exploração, na qual ocorreu a classificação e categorização do material;

- Tratamento e interpretação dos resultados obtidos.

Após a análise dos dados, foram obtidas duas categorias: conhecimento do enfermeiro sobre as terapias dialíticas e tratamento conservador: conhecimento e percepção do enfermeiro. Para manter a privacidade e confidencialidade dos participantes, decidiu-se não informar o sexo de cada sujeito, considerando o número reduzido de enfermeiros participantes da 
pesquisa e colaboradores no Hospital das Clínicas da UNICAMP, na Unidade de Internação Adulto (UIA), que poderiam ser facilmente identificáveis no local de estudo. Na apresentação das falas, optou-se por utilizar as letras "ENF" seguida do número correspondente à entrevista - por exemplo, à primeira entrevista foi designado o código ENF1.

Os dados obtidos na transcrição das entrevistas foram analisados com o auxílio do software QSR NVivo 11, versão Windows 19, para empregar a análise de conteúdo de Bardin (2009). O programa utilizado permitiu cruzar informações, codificar dados e gerenciar informações, assim como, ajudou na montagem das categorias temáticas

\section{Aspectos éticos}

O estudo não contou com a participação de grupos vulneráveis, sendo incluídos somente os indivíduos com idade maior ou igual a 18 anos e que demonstrarem condições físicas e cognitivas para responder e compreender o instrumento de coleta de dados, além da assinatura do TCLE.

Foi esclarecido aos enfermeiros participantes que o mesmo teria a liberdade para se recusar a responder qualquer item do questionário semiestruturado e retirar seu consentimento a qualquer momento, se assim desejasse, bem como seria garantida a confidencialidade dos dados e materiais obtidos, os quais são anônimos. Também foi comunicado aos participantes que não haveria riscos previsíveis quanto à dimensão física, psíquica, moral, intelectual, social, cultural ou espiritual do ser humano, em qualquer fase da pesquisa.

No momento do convite para participar da pesquisa, foi apresentado aos entrevistados o Termo de Consentimento Livre e Esclarecido (TCLE), com informações claras, objetivas e simples, sobre o intuito da pesquisa, garantia do sigilo e direito de exclusão em qualquer momento, sem qualquer prejuízo. A entrevista foi iniciada após a assinatura do TCLE, autorizando sua participação na pesquisa. O TCLE foi elaborado em duas vias, uma ficando com o pesquisador e uma com a participante da pesquisa, com o nome e telefone do responsável pela pesquisa.

Esta pesquisa foi submetida ao Comitê de Ética em Pesquisa do Centro Universitário da Fundação Hermínio Ometto / FHO e à Plataforma Brasil, sendo aprovada segundo CAAE $n^{\circ} 13710619.4 .0000 .5385$ e aprovação $n^{\circ}$ 3.374.048. As recomendações das Diretrizes e Normas para Pesquisa envolvendo Seres Humanos estabelecidas na Resolução 466/12 do Conselho Nacional de Saúde foram respeitadas.

\section{Resultados e Discussão}

Foram entrevistados dez enfermeiros, dos quais nove eram do sexo feminino e apenas um do sexo masculino. A faixa etária entre os participantes variou de 29 a 47 anos. Nenhum dos entrevistados possuía o título de enfermeiro nefrologista no período em que a pesquisa foi realizada. Contudo, todos relataram possuir experiência na assistência ao paciente portador de doença renal, em terapia de substituição renal e/ou em tratamento conservador.

Da análise das transcrições das entrevistas duas categorias temáticas emergiram: 1) conhecimento do enfermeiro sobre as terapias dialíticas e; 2) tratamento conservador: conhecimento e percepção do enfermeiro

\section{1) Conhecimento do enfermeiro sobre as terapias dialíticas}

Os resultados obtidos das entrevistas mostram que os enfermeiros possuem conhecimento sobre os tratamentos da doença renal crônica. Estes citaram mais frequentemente o tratamento hemodialítico, conforme pode ser observado nas falas a seguir: tratamento conservador, hemodiálise, diálise peritoneal e transplante renal (ENF 2); Sim, hemodiálise, medicações e alimentação (ENF 7); Hemodiálise, não dialítico, diálise peritoneal e transplante renal (ENF1 0).

Estima-se que a incidência anual de novos pacientes com doença renal em diálise, no Brasil, possa estar em torno de 42.500, com crescente tendência a aumentar. Calcula-se também que mais de 130 mil pacientes estejam em terapia de 
substituição renal, sendo que a hemodiálise é a mais prevalente. Segundo o censo brasileiro de diálise da Sociedade Brasileira de Nefrologia (SBN) em 2019, a distribuição de pacientes em hemodiálise (HD) foi um pouco mais de 93\%, enquanto o restante encontrava-se em diálise peritoneal (DP) (Neves, et al., 2020; Thomé, et al.; 2019). No ano anterior, segundo a Sociedade Brasileira de Nefrologia, a HD correspondeu à 89,9\% dos pacientes em TRS enquanto a DP foi responsável por 7,7\% desse quantitativo (Thomé, et al, 2019). Dentre as opções de tratamento dialítico, a hemodiálise é considerada como primeira escolha entre os profissionais que trabalham na assistência a pacientes com doença renal. Tal escolha, culturalmente enraizada no país, é acompanhada pela resistência a métodos alternativos à hemodiálise (Silva, Ribeiro, Ribeiro, Rosa \& CottaSilva, 2013). São os tratamentos que os pacientes fazem, como a hemodiálise. Aqui, eu assisto mais estes pacientes. (ENF3); como é um hospital universitário, temos todas as terapias de diálise, mas no setor há mais hemodiálise e depois temos a peritoneal. O transplante tem também (ENF8); temos mais hemodiálise, com certeza. Mas também tem o tratamento peritoneal e a conversa com o paciente sempre, né? (ENF9).

O conhecimento das terapias dialíticas revela que os enfermeiros reconhecem a hemodiálise como principal tratamento em detrimento da diálise peritoneal, transplante e tratamento conservador. A literatura reporta continuamente que somente a equipe médica acompanha o tratamento e as perspectivas dos pacientes em terapias dialíticas, em especial, a diálise peritoneal e o tratamento conservador (Bastos \& Kirsztajn, 2011).

Estima-se que aos demais integrantes da equipe multiprofissional, caiba a educação em saúde, a manutenção e o suporte, que são essenciais ao paciente durante a preparação para o início do tratamento. Como também, cabe à equipe multiprofissional toda a terapia, independente da modalidade escolhida e progressão da doença renal vivenciada. O tratamento completo e humanizado deste paciente em progressão e a escolha da TRS deve ser uma decisão conjunta, ocorrendo durante o tratamento conservador, além de ser discutido entre o paciente e a equipe de cuidados em saúde que o acompanha (Fernandes, et al., 2020; Bastos \& Kirsztajn, 2011).

Vale ressaltar que o sucesso do tratamento conservador traz consigo diversos fatores, sendo fundamental o envolvimento do paciente como o principal responsável pela redução da progressão da doença renal. O seguimento irrestrito às orientações prescritas pela equipe multiprofissional, a relação de confiança estabelecida entre todos os envolvidos - equipe/ paciente - os apoios familiar e social, aliadas às adequadas condições de alimentação e ingestão de medicamentos, traz consigo uma aceitação e qualidade de vida à este indivíduo, contribuindo, portanto, para o sucesso do tratamento e melhor percepção da terapia dialítica em andamento (Fernandes, et al., 2020; Lima, Paula, Duarte, Magro \& Lima, 2020).

Os pacientes portadores de doença renal demonstram uma resistência às demais terapias dialíticas quando há envolvimento da substituição renal. Acredita-se que a falta de orientação profissional e a fragmentação do cuidado, em especial na Atenção Primária à Saúde (APS), pelas Estratégias de Saúde da Família, tem contribuído para a manutenção dessa resistência. Contudo, ressalta-se que ações programáticas na APS, com verificação das principais doenças de base que influenciam na decorrência da doença renal e sua progressão, assim como o encaminhamento precoce ao nefrologista, trazem benefícios diretos aos usuários (Lima, et al., 2020).

Estas verificações contribuem para a sobrevida com qualidade, reduzindo assim as complicações que a progressão da doença ocasiona, e melhoram o conhecimento das formas de terapia dialítica quando já iniciada a perda da função renal (Cunha, Harzheim, Medeiro, D'Avila, Martins, Wollmann \& Faller, 2020). Segundo Lima, et al. (2020), o conhecimento insuficiente sobre as terapias dialíticas e a estratificação da progressão da doença renal na APS é reconhecido como fator limitante para elaboração estratégica de medidas intervenientes no processo de tomada de decisão. Estas estratégias, podem conduzir uma mudança real na melhoria da saúde dos usuários, possibilitando que reconheçam os principais fatores de risco da doença renal (Lima, et al., 2020). 
O enfermeiro destaca-se na qualificação da equipe de cuidados em diálise, ganhando um papel central no emprego das tecnologias, de educação em saúde, através da difusão de conhecimento sobre o tratamento conservador junto aos pacientes (Cunha, et al., 2020; Mendes, Alves, Bucuvi, Dias, Ponce, Mendes \& Alves, 2017). Logo, a falta de conhecimento sobre a doença renal, sua progressão, manejo clínico e terapias dialíticas, pode contribuir para que o enfermeiro sinta insegurança na condução das mais diversas orientações sobre a ações no tratamento conservador, mantendo, portanto, a cultura do tratamento hemodialítico com maior frequência entre as terapias disponíveis (Chaves; Borges, Silva \& Gomes, 2017). Por fim, conhecer as terapias dialíticas pelos enfermeiros que atuam na assistência aos portadores de doença renal é fundamental para direcionar estratégias de implantação de melhorias na assistência à saúde, assim como reconhecer as progressões e tratamentos possíveis para a perda da função renal potencializa medidas transformadoras na rotina dos pacientes (Corrêa, Araújo, Ribeiro \& Pedrosa, 2012).

\section{2) Tratamento conservador: conhecimento e percepção do enfermeiro}

A amostra entrevistada demonstrou conhecer o tratamento conservador como um processo de redução da progressão da perda da função renal, associando-o como um dos cuidados prestados pelo enfermeiro. [...] O tratamento conservador ajuda retardar esse processo, porém não evita a falência do órgão (ENF2); [...]consiste em todas as medidas clínicas (remédios, mudança da dieta e estilo de vida) para reduzir a velocidade e a progressão da doença, principalmente nos pacientes idosos (ENF5); o tratamento conservador é quando o paciente tem que fazer as restrições, principalmente na dieta, para não perder mais rápida a função do rim (ENF6).

O tratamento conservador consiste em uma opção pré-terapia dialítica que associa um conjunto de medidas que visam reduzir a progressão da perda da função renal, através de ações que melhoram as condições clínicas, físicas, sociais e psicológicas dos portadores (CDC, 2019). Este tipo de tratamento está associado à Atenção Primária à Saúde, que ao promover a saúde, pode também prevenir agravos nos grupos de risco ao identificar precocemente os estágios da doença e detectar a diminuição da taxa de filtração glomerular (TFG). Tais conjuntos de medidas podem contribuir para corrigir as causas reversíveis da insuficiência renal e auxiliar no planejamento precoce a fim de evitar a TRS (Canziani \& Kirsztajn, 2017; Brasil, 2014).

A literatura traz resultados positivos entre aqueles indivíduos em tratamento conservador e que possuem o enfermeiro como agente de ensino, principalmente sobre o autocuidado. O autocuidado por sua vez, é conquistado por ações de educação em saúde proporcionadas pelas consultas de enfermagem, que levantam as necessidades do paciente e buscam estratégias eficazes, desenvolvendo conjuntamente, as competências necessárias às mudanças advindas com a progressão da doença renal, assim como empoderando o portador sobre sua condição de cronicidade. Através do conhecimento transmitido, o enfermeiro estimula a conservação da função renal, ao oferecer informações e instruções sobre as adaptações fundamentais, como o uso correto das medicações, a execução da atividade física compatível com a condição clínica, a orientação da alimentação balanceada e até mesmo o lazer, podendo determinar, portanto, a saúde destes pacientes (Chehuen Neto, Costa, Estevanin, Bignoto, Vieira, Pinto \& Ferreira, 2019).

Também se verificou que um integrante da pesquisa percebe o tratamento conservador como o princípio de toda a terapia dialítica. Enquanto outro entrevistado, reconhece tal tratamento como uma fase pelo qual portadores de doenças crônicas podem vivenciar, como a negação: o tratamento conservador é o primeiro tratamento para evitar a hemodiálise (ENF10); o tratamento é utilizado para pacientes em início de doença ou que se negam a realizar a hemodiálise ou diálise peritoneal (ENF9).

Em 2019, segundo o censo da Sociedade Brasileira de Nefrologia, foram identificados 42,5\% dos pacientes em diálise com idades entre 45 a 64 anos de idade, 22,2\% com idades de 65 a 74 anos e 13,3\% daqueles com idade de 75 e mais (Neves, et al., 2020). Estes dados de frequência corroboram o trabalho de Castro (2019), no qual apresenta uma tendência de realizar a 
hemodiálise em pacientes idosos, particularmente em indivíduos acima de 70 anos de idade. O mesmo estudo, no entanto, também traz a discussão acerca do tratamento conservador como uma opção não invasiva e mais branda diante da doença instaurada, já que grande parte deste público apresenta maior número de comorbidades e fatores associados que podem contribuir para a sua mortalidade.

O curso crônico e as diferentes fases vivenciadas por portadores de doença renal crônica puderam ser verificados por Pichinelli \& Milagres (2018), em um trabalho sobre a percepção da insuficiência renal crônica e o enfrentamento de pacientes jovens em tratamento hemodialítico. Segundo as autoras, apesar dos jovens vivenciarem anteriormente negação e revolta, principais fases no princípio da doença, demonstram, já em última fase no momento da pesquisa realizada, aceitação, demonstrando um otimismo em relação à vida e sua continuidade.

$\mathrm{Na}$ assistência de enfermagem ao portador de doença renal na Unidade de Internação Hospitalar, os enfermeiros que relataram cuidar de portadores de doença renal em terapia conservadora, mencionaram que os indivíduos, em sua maioria apresentavam faixa etária superior a 70 anos. A vivência deste tratamento conservador não foi observada em três integrantes desta pesquisa. Os demais, apesar da experiência e conhecendo as orientações dos hábitos de vida modificáveis, já fundamentadas pela literatura, relataram dificuldades na adesão pelo paciente. [...] realizava consulta de enfermagem e orientava aos pacientes quanto a controlar hipertensão e diabetes, realizar atividade física, diminuir ingestão de proteínas, fazer uso de diuréticos conforme orientação médica, aumentar ou reduzir ingestão de líquidos (ENF1); [...]já realizei cuidado de vários pacientes com tratamento conservador, a maioria eram idosos em início da doença renal (ENF9); sempre vejo que orientar a mudança de alimentação é a parte mais difícil porque eles não querem mudar. A atividade física também é complicada, ainda mais para aqueles com mais idade (ENF10).

Muitos pacientes conseguem se adequar ao tratamento conservador, interferindo positivamente na percepção da doença e na estabilidade ou melhora de sinais e sintomas da perda da função renal. Esta percepção do paciente pode ser alcançada através das mudanças de hábitos de vida, no qual as modificações nos hábitos alimentares e na prática de exercícios físicos estão entre as mais prevalentes. No entanto, o doente renal, muitas vezes precisa de apoio para seguir as orientações disponibilizadas pelo enfermeiro, devendo este, continuamente, reavaliar as condições de adesão e de mudanças de hábitos modificáveis de vida, propostos pelo tratamento conservador (Castro, 2019).

Durante a entrevista os enfermeiros relataram conseguir identificar a adesão ao tratamento conservador pelos pacientes. No entanto, para melhor realizarem orientações, reconhecem que é necessária prévia capacitação para conhecimento e entendimento de tal terapia: [...] se o paciente segue corretamente as orientações acredito que consiga melhorar um pouco seu quadro e retardar medidas mais intervencionistas (ENF1); [...] a eficácia desse tipo de tratamento está ligada ao nível de comprometimento do paciente (ENF6); [...] se houver uma adesão desde o início o paciente consegue estender por muito tempo o tratamento da doença sem precisar de diálise (ENF9).

Da amostra entrevistada, a maioria alegou não possuir especialização em nefrologia para assistir ao paciente portador de doença renal crônica, sentindo-se inseguros para orientar acerca do tratamento conservador. Contudo, relatam que o auxílio por parte da equipe multiprofissional do setor de nefrologia do referido hospital, proporciona mais propriedade e mais segurança para praticar tal terapia junto aos pacientes que necessitam. A falta de conhecimento pelo enfermeiro e demais profissionais de saúde interfere diretamente na construção de cuidados à saúde. Vale lembrar que conhecimento e capacitação são partes integrantes das diversas mudanças nos hábitos de vida do indivíduo portador de DRC e dos profissionais que o atendem (Ribeiro, Andrade, Fassarella, Santana, Silva Costa \& Morais, 2018)

Assim relatam os enfermeiros: [...] sozinha não oriento o tratamento de diálise, nem mesmo dessa terapia conservadora. Eu dependo de ações do conjunto de nós todos aqui do hospital e de outras profissões (ENF 2); [...] se eu tivesse uma capacitação profissional adequada para essa função, sim, me sentiria segura. Mas temos um suporte do pessoal da 
nefrologia que ajuda bem (ENF 6); [...]se fosse realizado por equipe multidisciplinar com pessoas especializas e com experiência (ENF10).

Quanto à insegurança dos enfermeiros em orientar a terapia dialítica conservadora, uma capacitação específica é possível, através da implantação de planos de cuidados criados por profissionais da nefrologia a estes enfermeiros inseridos em setores de internação hospitalar. A partir daí a propagação das medidas que proporcionam entendimento e independência dos pacientes pode ser alcançada, deixando-os mais atuantes no controle do seu tratamento e de sua qualidade de vida (Fernandes et al., 2020; Roso, Beuter, Jacobi, Silva, Perrando \& Bruinsma, 2013). [...] Cuidar da parte psicológica é tão importante quanto da parte física em si, acredito que montar um grupo terapêutico de pacientes renais crônicos auxiliaria principalmente na adesão ao tratamento, além dos controles de outras doenças crônicas como a hipertensão e o diabetes (ENF2); [...]basicamente orientação verbal quanto ao entendimento e avaliação da adesão (ENF8); [...] orientações, grupos de apoio, consultas de enfermagem com exame físico rigoroso, acompanhamento com familiares (ENF9).

O enfermeiro deve ser o profissional capaz de acompanhar um portador de doença renal, orientando-os corretamente, assim como aos seus familiares e responsáveis. A implantação do tratamento conservador orientado, o mais precocemente possível, preferencialmente durante o primeiro contato com a doença e aplicado como uma terapia de escolha para a maioria dos casos iniciais, auxilia em melhores desfechos. Esta terapia leva em consideração a menor complexidade, melhora da qualidade de vida do indivíduo e menor custo-benefício (Fernandes et al., 2020).

No entanto, o despreparo dos profissionais e a visão de um tratamento que não seja invasivo, remete a estes profissionais, uma percepção de ineficácia deste tratamento. Desta forma, profissionais que não apresentam interesse nas diversas áreas em que a nefrologia atua, pode ser um agente desmotivador da terapia conservadora, uma vez que não estimulam os usuários a adequarem-se ao novo estilo de vida imposto pela doença renal (Milagres \& Santos, 2012).

Este trabalho apresenta algumas limitações. As entrevistas foram realizadas em apenas um setor do hospital universitário e apresentou um número reduzido de sujeitos pesquisados, podendo não refletir a realidade sobre o conhecimento e a percepção deste profissional quanto ao tratamento conservador em portadores de doença renal. Os enfermeiros entrevistados não possuíam especialização em nefrologia e apresentaram reduzido conhecimento sobre cuidados em saúde específicos da progressão da perda da função renal.

Contudo, apesar destas limitações e do delineamento transversal, a pesquisa demonstrou que este é um profissional que se faz presente no cotidiano de indivíduos com doença renal, devendo continuar buscando conhecimento e capacitação dos cuidados específicos do estadiamento desta doença crônica, assim como reconhecendo as mudanças impostas aos seus portadores. Tais condutas permitem ao enfermeiro proporcionar mais segurança ao paciente frente ao tratamento dialítico.

\section{Conclusão}

A DRC possui caráter insidioso com alta penetração no cenário mundial. Assim, o tratamento conservador pode auxiliar no processo de manutenção da função renal por tempo mais prolongado. Dentro desse panorama, demonstrar como é a visão e conhecimento do enfermeiro frente ao paciente renal que está em terapia substitutiva ou conservadora, afirmou a grande necessidade de capacitação permanente em todos os setores hospitalares e na atenção básica à saúde. Pois, somente a partir da compreensão do papel de cada membro da equipe multiprofissional e do paciente, mudanças podem ser geradas. Devido ao paciente renal dialítico e conservador estar em todos os setores de uma instituição hospitalar, é imprescindível que todos os setores compreendam como prestar um serviço de qualidade a este público, não limitando as especificidades apenas aos profissionais do setor de nefrologia.

Os dados obtidos puderam demonstrar a importância da educação em saúde que podem ser obtidos através de maiores investimentos formativos dos profissionais e equipes que trabalham com pacientes em tratamento conservador. Tais 
investimentos fazem-se importantes pois promovem e possibilitam empoderamento dos pacientes frente à doença. Trabalhos futuros em diferentes centros que realizam tratamento conservador podem se beneficiar destes dados, assim como gestores de saúde que buscam melhor qualidade de vida aos usuários nos mais diversos níveis de complexidade em saúde que o sistema atende.

\section{Referências}

Bardin, L, (2009), Análise de conteúdo, Lisboa, Edições 70 LDA,

Bastos, M, G, \& Kirsztajn, G, M, (2011), Doença renal crônica: importância do diagnóstico precoce, encaminhamento imediato e abordagem interdisciplinar estruturada para melhora do desfecho em pacientes ainda não submetidos à diálise, Brazilian Journal of Nephrology 33 (1): 93-108, doi,org/10,1590/S010128002011000100013 ,

Brasil, Ministério da Saúde, Secretaria de Atenção à Saúde, Departamento de Atenção Especializada e Temática (2014), Diretrizes clínicas para o cuidado ao paciente com doença renal crônica - Doença renal crônica no Sistema Único de Saúde, p, 37 ,

Canziani, M, F, \& Kirsztajn, G, M, (2017), Doença renal crônica: manual prático uso diário, ambulatorial e hospitalar, (2a ed.), Balieiro,

Castro, M, C, M, (2019), Conservative management for patients with chronic kidney disease refusing dialysis, Brazilian Journal of Nephrology 41: 95-102, doi,org/10,1590/2175-8239-jbn-2018-0028

Centers for Disease Control and Prevention - CDC (2019), Chronic kidney disease in the United States, Atlanta, GA: US Department of Health and Human Services, Centers for Disease Control and Prevention,

Chaves, E, A., Borges, A, S., Silva, P, C, \& Gomes, C, T, (2017), Atuação do enfermeiro frente ao paciente em diálise peritoneal: revisão integrativa da literatura, Revista Renome 6 (2): 48-59,

Chehuen Neto, J, A., Costa, L, A., Estevanin, G, M., Bignoto, T, C., Vieira, C, I, R., Pinto, F, A, R., Ferreira, R, E, (2019), Functional health literacy in chronic cardiovascular patients, Ciência \&amp; Saúde Coletiva 24 (3): 1121-32, doi,org/10,1590/1413-81232018243,02212017,

Corrêa, Á, C, P., Araújo, E, M; Ribeiro, A, C, \& Pedrosa, I, C, F, (2012), Perfil sociodemográfico e profissional dos enfermeiros da atenção básica à saúde de Cuiabá - Mato Grosso, Revista Eletrônica de Enfermagem 14 (1):171-180, doi,org/10,5216/ree,v14i1,12491,

Cunha, C, R, H., Harzheim, E., Medeiro, O, L, D'Avila,O, P., Martins, C., Wollmann, L., \& Faller, L, A, (2020), Primary health care portfolio: assuring of integrality in the family health and oral health teams in Brazil, Ciência \& Saúde Coletiva 25 (4):1313-26,doi,org/10,1590/1413-81232020254,31862019,

Daugirdas, J, T., Blake, P, G., \& Todd, S, (2016). (5a ed.) Guanabara Koogan,

Fernandes, D., Zanelli, T, L, P., Scharr Rodrigues, A., Rodrigues, M, P., Lodi, J, C., Marques, T, M., Reis, G, Ferreira, L, R, C, \& Milagres, C, S, (2020), Qualidade de vida de pacientes em terapia renal substitutiva: uma análise da doença renal crônica e perfil populacional de isco, Revista Eletrônica Acervo Saúde 12 (12): e4759-e4759, doi,org/10,25248/reas,e4759,2020,

Gonçalves, F, A., Dalosso, I, F., Borba, J, M, C., Bucaneve, J., Valerio, N, M, P., Okamoto, C, T, \& Bucharles, S, G, E, (2015), Quality of life in chronic renal patients on hemodialysis or peritoneal dialysis: a comparative study in a referral service of Curitiba - PR, Jornal Brasileiro de Nefrologia 37 (4): $467-$ 74,doi,org/10,5935/0101-2800,20150074,

Lima, W, L., Paula, L, B., Duarte, T, P., Magro, M, C, S, \& Lima, W, L, (2020), Conhecimento dos enfermeiros da atenção primária à saúde sobre fatores de risco para Lesão Renal Aguda, Escola Anna Nery 24 (2), e20190280, Epub February 27,2020, doi,org/10,1590/2177-9465-ean-2019-0280,

Mendes, M, L., Alves, C, A., Bucuvi, E, M., Dias, D, B; Ponce, D., Mendes, M, L, \& Alves, C, A, (2017), Diálise peritoneal como primeira opção de tratamento dialítico de início não planejado, Brazilian Journal of Nephrology 39 (4): 441-46, doi,org/10,5935/0101-2800,20170077,

Milagres, C, S, \& Santos, L, C, G, (2012), Unveiling the nephrological nurse's universe in the hemodialysis units, Rev enferm UFPE on line, Aug;6(8):18719,doi,org/10,5205/1981-8963-V6I8A7280P1871-1879-2012,

Moura Neto, J, Á., Souza, A, F, P, Moura, D, Q., Oliveira, G, M, Paschoalim, S, P., Paschoalim, E, L, \& Moura Júnior, J, A, (2014), Modalidade de terapia renal substitutiva como preditora de sintomas depressivos, Jornal Brasileiro de Psiquiatria 63 (4): 354-59, doi,org/10,1590/0047-2085000000045,

Neves, P, D, M, M., Sesso, R, C, C, Thomé, F, S., Lugon, J, R, \& Nascimento, M, M, (2020), Brazilian dialysis census: analysis of data from the 2009-2018 decade, Brazilian Journal of Nephrology, 42(2), 191-200. doi,org/10,1590/2175-8239-jbn-2019-0234,

Pereira, E., Chemin, J., Menegatti, C, L, \& Riella, M, C, (2016), Escolha do método dialítico - variáveis clínicas e psicossociais relacionadas ao tratamento, Brazilian Journal of Nephrology (Jornal Brasileiro de Nefrologia) 38 (2):215-24, doi,org/10,5935/0101-2800,20160031,

Pereira, R, M, P., Batista, M, A., Meira, A, S., Oliveira, M, P, \& Kusumota, L, (2017), Quality of life of elderly people with chronic kidney disease in conservative treatment, Revista Brasileira de Enfermagem 70 (4): 851-59, doi,org/10,1590/0034-7167-2017-0103,

Pereira, A, S., Shitsuka, D, M., Parreira, F, J., \& Shitsuka, R. (2018), Metodologia da pesquisa científica. UFSM.

Pichinelli, J, J, \& Milagres, C, S, (2018), Percepção da insuficiência renal crônica e enfrentamento de pacientes jovens em tratamento hemodialítico, Enfermagem Brasil 17 (3): 182-89, doi,org/10,33233/eb,v17i3,1177, 
Research, Society and Development, v. 10, n. 2, e5310212237, 2021

(CC BY 4.0) | ISSN 2525-3409 | DOI: http://dx.doi.org/10.33448/rsd-v10i2.12237

Ribeiro, W, A., Andrade, M., Fassarella, B, P, A., Santana, P, P, C., Silva Costa, P. A, F, \& Morais, M, C, (2018), Enfermeiro protagonista na educação em saúde para o autocuidado de pacientes com doenças renal crônica, Revista Pró-UniverSUS 9 (2): 60-65,

Roso, C, C., Beuter, M., Jacobi, C, S., Silva, C, T., Perrando, M, S, \& Bruinsma, J, L, (2013), Progressão da insuficiência renal crônica: percepções de pessoas em pré-diálise, Revista de Enfermagem da UFSM 3 (0): 581-88, doi,org/10,5902/2179769211020,

Silva, S., Ribeiro, R, C., Ribeiro, L; Rosa, C, O, B, \& Cotta, R, M, M, (2013), Tratamento conservador: influência sobre parâmetros clínicos de indivíduos em hemodiálise, Mundo saúde (1995), 354-64,

Thomé, F, S., Sesso, R, C., Lopes, A, A., Lugon, J, R, \& Martins, C, T, (2019), Brazilian chronic dialysis survey 2017, Brazilian Journal of Nephrology, 41 (2), 208-214, Epub 28 de março de 2019. doi,org/10,1590/2175-8239-jbn-2018-0178,

Tong, A; Sainsbury, T, \& Craig, T, (2007), Consolidated Criteria for Reporting Qualitative Research (COREQ): A 32-Item checklist for interviews and focus groups, International Journal for Quality in Health Care: Journal of the International Society for Quality in Health Care 19 (6): 349-57, doi,org/10,1093/intqhe/mzm042,

Xavier, B, L, S., Hermógenes, J, F, A., Ribeiro, Y, C., Sá, A, C, S., Ávila, F. M. V., \& Flores, P, V, P, (2020), Senses and meanings of conservative treatment in people with chronic kidney disease, Aquichan, 20 (3), e2035, doi,org/10,5294/aqui,2020,20,3,5 